\title{
Improving Respiratory Rate Accuracy in the Hospital: A Quality Improvement Initiative
}

\author{
Neil Keshvani, MD*, Kimberly Berger, MD, MPH ${ }^{1}$, Arjun Gupta, MD¹, Sheila DePaola, RN², \\ Oanh Kieu Nguyen, MD, MAS ${ }^{1,3}$, Anil N Makam, MD, MAS 1,3
}

'Department of Internal Medicine, UT Southwestern Medical Center, Dallas, Texas; ${ }^{2}$ Department of Nursing, Parkland Health and Hospital System, Dallas, Texas; ${ }^{3}$ Department of Medicine, Chan Zuckerberg San Francisco General Hospital, University of California, San Francisco, California.

Respiratory rate $(\mathrm{RR})$ is a predictor of adverse outcomes. However, RRs are inaccurately measured in the hospital. We conducted a quality improvement (Ql) initiative using plan-do-study-act methodology on one inpatient unit of a safety-net hospital to improve RR accuracy. We added time-keeping devices to vital sign carts and retrained patient-care assistants on a newly modified workflow that included concomitant RR measurement during automated blood pressure measurement. The median RR was 18 (interquartile range [IQR] 18-20) preintervention versus 14 (IQR 15-20) postintervention. RR accuracy, defined as \pm 2 breaths of gold-standard measurements, increased from $36 \%$ preintervention to $58 \%$ postintervention $(P<.01)$. The median time for vital signs decreased from 2:36 minutes (IQR, 2:04-3:20) to 1:55 minutes (IQR, 1:40-2:22; $P<.01$ ). The intervention was associated with a $7.8 \%$ reduced incidence of tachypnea-specific systemic inflammatory response syndrome (SIRS $=2$ points with $\mathrm{RR}>20 ; 95 \% \mathrm{Cl}$, $-13.5 \%$ to $-2.2 \%)$. Our interdisciplinary, low-cost, low-tech Ql initiative improved the accuracy and efficiency of RR measurement. Journal of Hospital Medicine 2019;14:673677. (C) 2019 Society of Hospital Medicine

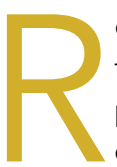

espiratory rate (RR) is an essential vital sign that is routinely measured for hospitalized adults. It is a strong predictor of adverse events. ${ }^{1,2}$ Therefore, RR is a key component of several widely used risk prediction scores, including the systemic inflammatory response syndrome (SIRS). ${ }^{3}$

Despite its clinical utility, RR is inaccurately measured. ${ }^{4-7}$ One reason for the inaccurate measurement of RR is that RR measurement, in contrast to that of other vital signs, is not automated. The gold-standard technique for measuring RR is the visual assessment of a resting patient. Thus, RR measurement is perceived as time-consuming. Clinical staff instead frequently approximate RR through brief observation. ${ }^{8-11}$

Given its clinical importance and widespread inaccuracy, we conducted a quality improvement (QI) initiative to improve RR accuracy.

\section{METHODS}

\section{Design and Setting}

We conducted an interdisciplinary $\mathrm{Q}$ initiative by using the plan-do-study-act (PDSA) methodology from July 2017 to February 2018. The initiative was set in a single adult 28-bed medical inpatient unit of a large, urban, safety-net hospital

*Corresponding Author: Neil Keshvani, MD; E-mail: Neil.Keshvani@gmail.com; Telephone: 214-648-2287; Twitter: @NeilKeshvani.

Published online first June 10, 2019

Find Additional Supporting Information in the online version of this article.

Received: February 24, 2019; Revised: April 11, 2019; Accepted: April 22, 2019

(c) 2019 Society of Hospital Medicine DOI 10.12788/jhm.3232 consisting of general internal medicine and hematology/oncology patients. Routine vital sign measurements on this unit occur at four- or six-hour intervals per physician orders and are performed by patient-care assistants (PCAs) who are nonregistered nursing support staff. PCAs use a vital signs cart equipped with automated tools to measure vital signs except for RR, which is manually assessed. PCAs are trained on vital sign measurements during a two-day onboarding orientation and four to six weeks of on-the-job training by experienced PCAs. PCAs are directly supervised by nursing operations managers. Formal continuing education programs for PCAs or performance audits of their clinical duties did not exist prior to our Ql initiative.

\section{Intervention}

Intervention development addressing several important barriers and workflow inefficiencies was based on the direct observation of PCA workflow and information gathering by engaging stakeholders, including PCAs, nursing operations management, nursing leadership, and hospital administration (PDSA cycles 1-7 in Table). Our modified PCA vital sign workflow incorporated RR measurement during the approximate 30 seconds needed to complete automated blood pressure measurement as previously described. ${ }^{12}$ Nursing administration purchased three stopwatches (each $\$ 5$ US) to attach to vital signs carts. One investigator (NK) participated in two monthly one-hour meetings, and three investigators (NK, KB, and SD) participated in 19 daily 15-minute huddles to conduct stakeholder engagement and educate and retrain PCAs on proper technique (total of 6.75 hours). 
TABLE. PDSA Cycles for the Design and Implementation of a Quality Improvement Initiative to Improve Respiratory Rate Accuracy

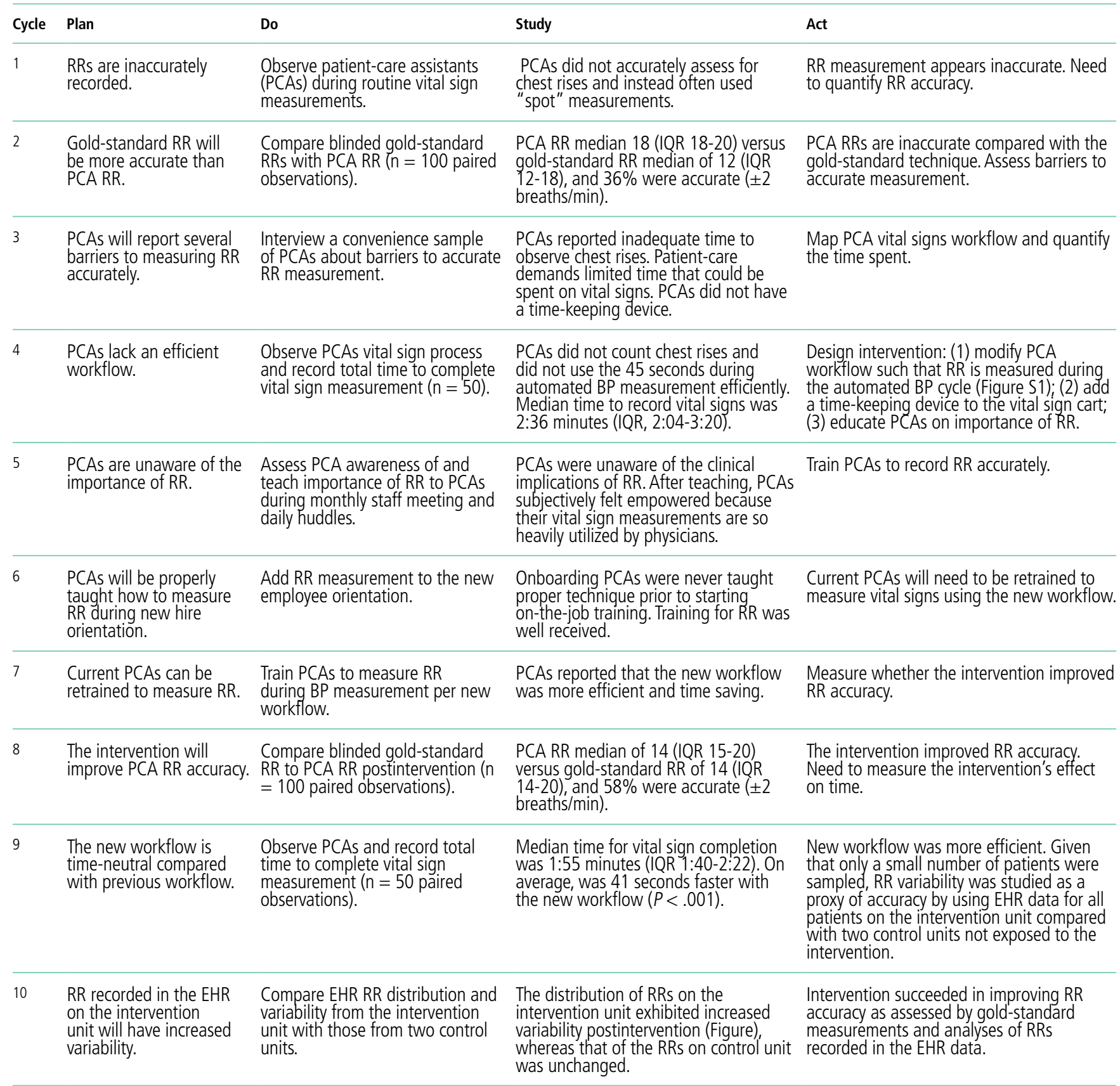

Abbreviations: EHR, electronic health record; IQR, interquartile range; PCA, patient-care assistants; PDSA, plan-do-study-act; RR, respiratory rate.

\section{Evaluation}

The primary aim of this Ql initiative was to improve RR accuracy, which was evaluated using two distinct but complementary analyses: the prospective comparison of PCA-recorded RRs with gold-standard recorded RRs and the retrospective comparison of RRs recorded in electronic health records (EHR) on the intervention unit versus two control units. The secondary aims were to examine time to complete vital sign measurement and to assess whether the intervention was associated with a reduction in the incidence of SIRS specifically due to tachypnea.

\section{Respiratory Rate Accuracy}

PCA-recorded RRs were considered accurate if the RR was within \pm 2 breaths of a gold-standard RR measurement performed by a trained study member (NK or KB). We conducted gold-standard RR measurements for 100 observations preand postintervention within 30 minutes of PCA measurement 


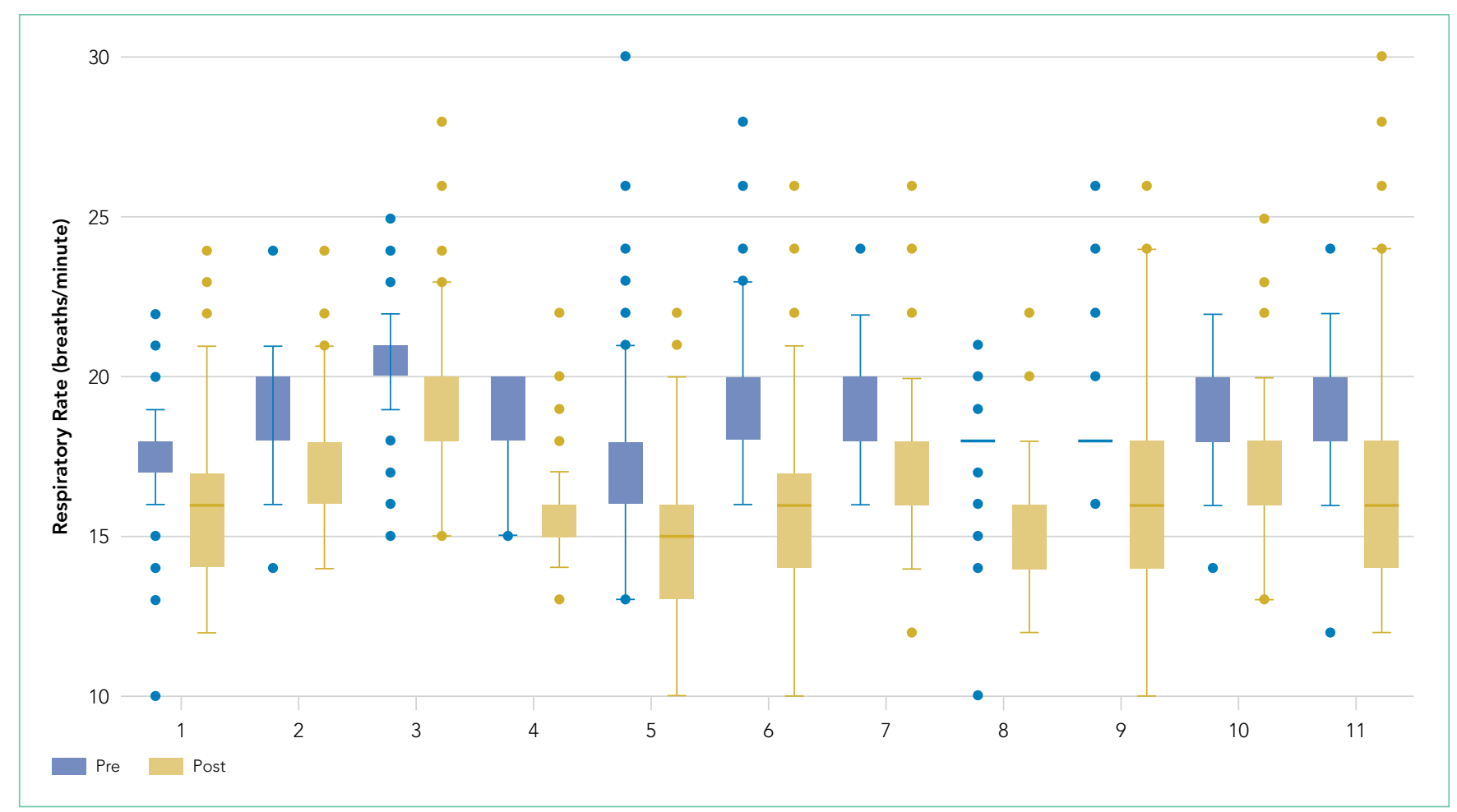

FIG. Distribution of Recorded Respiratory Rates by 11 Individual PCAs Pre- and Postintervention. ${ }^{a}$

alndividual PCAs were included if they recorded $\geq 100$ respiratory rates during the postintervention period. Abbreviations: PCA, patient-care assistant

to avoid Hawthorne bias.

We assessed the variability of recorded RRs in the EHR for all patients in the intervention unit as a proxy for accuracy. We hypothesized on the basis of prior research that improving the accuracy of RR measurement would increase the variability and normality of distribution in RRs. ${ }^{13}$ This is an approach that we have employed previously. ${ }^{7}$ The EHR cohort included consecutive hospitalizations by patients who were admitted to either the intervention unit or to one of two nonintervention general medicine inpatient units that served as concurrent controls. We grouped hospitalizations into a preintervention phase from March 1, 2017-July 22, 2017, a planning phase from July 23, 2017-December 3, 2017, and a postintervention phase from December 21, 2017-February 28, 2018. Hospitalizations during the two-week teaching phase from December 3, 2017-December 21, 2017 were excluded. We excluded vital signs obtained in the emergency department or in a location different from the patient's admission unit. We qualitatively assessed RR distribution using histograms as we have done previously.

We examined the distributions of RRs recorded in the EHR before and after intervention by individual PCAs on the intervention floor to assess for fidelity and adherence in the PCA uptake of the intervention.

\section{Time}

We compared the time to complete vital sign measurement among convenience samples of 50 unique observations preand postintervention using the Wilcoxon rank sum test.

\section{SIRS Incidence}

Since we hypothesized that improved RR accuracy would reduce falsely elevated RRs but have no impact on the other three SIRS criteria, we assessed changes in tachypnea-specific SIRS incidence, which was defined a priori as the presence of exactly two concurrent SIRS criteria, one of which was an elevated RR. ${ }^{3}$ We examined changes using a difference-in-differences approach with three different units of analysis (per vital sign measurement, hospital-day, and hospitalization; see footnote for Appendix Table 1 for methodological details. All analyses were conducted using STATA 12.0 (StataCorp, College Station, Texas).

\section{RESULTS}

\section{Respiratory Rate Accuracy}

Prior to the intervention, the median PCA RR was 18 (IQR 1820) versus 12 (IQR 12-18) for the gold-standard RR (Appendix Figure 1), with only $36 \%$ of PCA measurements considered accurate. After the intervention, the median PCA-recorded RR was 14 (IQR 15-20) versus 14 (IOR 14-20) for the gold-standard RR and a RR accuracy of $58 \%(P<.001)$.

For our analyses on RR distribution using EHR data, we included 143,447 unique RRs (Appendix Table 2). After the intervention, the normality of the distribution of RRs on the intervention unit had increased, whereas those of RRs on the control units remained qualitatively similar pre- and postintervention (Appendix Figure 2).

Notable differences existed among the 11 individual PCAs 
(Figure) despite observing increased variability in PCA-recorded RRs postintervention. Some PCAs (numbers 2, 7, and 10) shifted their narrow RR interquartile range lower by several breaths/minute, whereas most other PCAs had a reduced median RR and widened interquartile range.

\section{Time}

Before the intervention, the median time to complete vital sign measurements was 2:36 (IQR 2:04-3:20). After the intervention, the time to complete vital signs decreased to 1:55 (IQR, 1:402:22; $P<.001)$, which was 41 less seconds on average per vital sign set.

\section{SIRS Incidence}

The intervention was associated with a 3.3\% reduction $(95 \%$ $\mathrm{Cl},-6.4 \%$ to $-0.005 \%$ ) in tachypnea-specific SIRS incidence per hospital-day and a $7.8 \%$ reduction $(95 \% \mathrm{Cl},-13.5 \%$ to $-2.2 \%)$ per hospitalization (Appendix Table 1). We also observed a modest reduction in overall SIRS incidence after the intervention (2.9\% less per vital sign check, $4.6 \%$ less per hospital-day, and $3.2 \%$ less per hospitalization), although these reductions were not statistically significant.

\section{DISCUSSION}

Our Ql initiative improved the absolute RR accuracy by $22 \%$, saved PCAs 41 seconds on average per vital sign measurement, and decreased the absolute proportion of hospitalizations with tachypnea-specific SIRS by $7.8 \%$. Our intervention is a novel, interdisciplinary, low-cost, low-effort, low-tech approach that addressed known challenges to accurate RR measurement, 8,9,11 as well as the key barriers identified in our initial PDSA cycles. Our approach includes adding a time-keeping device to vital sign carts and standardizing a PCA vital sign workflow with increased efficiency. Lastly, this intervention is potentially scalable because stakeholder engagement, education, and retraining of the entire PCA staff for the unit required only 6.75 hours.

While our primary goal was to improve RR accuracy, our Ql initiative also improved vital sign efficiency. By extrapolating our findings to an eight-hour PCA shift caring for eight patients who require vital sign checks every four hours, we estimated that our intervention would save approximately 16:24 minutes per PCA shift. This newfound time could be repurposed for other patient-care tasks or could be spent ensuring the accuracy of other vital signs given that accurate monitoring may be neglected because of time constraints. ${ }^{11}$ Additionally, the improvement in RR accuracy reduced falsely elevated RRs and thus lowered SIRS incidence specifically due to tachypnea. Given that EHR-based sepsis alerts are often based on SIRS criteria, improved RR accuracy may also improve alarm fatigue by reducing the rate of false-positive alerts. ${ }^{14}$

This initiative is not without limitations. Generalizability to other hospitals and even other units within the same hospital is uncertain. However, because this initiative was conducted within a safety-net hospital, we anticipate at least similar, if not increased, success in better-resourced hospitals. Second, the long-term durability of our intervention is unclear, although EHR RR variability remained steady for two months after our intervention (data not shown).

To ensure long-term sustainability and further improve RR accuracy, future PDSA cycles could include electing a PCA "vital signs champion" to reiterate the importance of RRs in clinical decision making and ensure adherence to the modified workflow. Nursing champions act as persuasive change agents that disseminate and implement healthcare change, ${ }^{15}$ which may also be true of PCA champions. Additionally, future PDSA cycles can obviate the need for labor-intensive manual audits by leveraging EHR-based auditing to target education and retraining interventions to PCAs with minimal RR variability to optimize workflow adherence.

In conclusion, through a multipronged $\mathrm{Q}$ initiative we improved RR accuracy, increased the efficiency of vital sign measurement, and decreased SIRS incidence specifically due to tachypnea by reducing the number of falsely elevated RRs. This novel, low-cost, low-effort, low-tech approach can readily be implemented and disseminated in hospital inpatient settings.

\section{Acknowledgments}

The authors would like to acknowledge the meaningful contributions of Mr. Sudarshaan Pathak, RN, Ms. Shirly Koduvathu, RN, and Ms. Judy Herrington MSN, RN in this multidisciplinary initiative. We thank Mr. Christopher McKintosh, RN for his support in data acquisition. Lastly, the authors would like to acknowledge all of the patient-care assistants involved in this $\mathrm{Ql}$ initiative.

Disclosures: Dr. Makam reports grants from NIA/NIH, during the conduct of the study. All other authors have nothing to disclose.

Funding: This work is supported in part by the Agency for Healthcare Research and Quality-funded UT Southwestern Center for Patient-Centered Outcomes Research (R24HS022418). OKN is funded by the National Heart, Lung, and Blood Institute (K23HL133441), and ANM is funded by the National Institute on Aging (K23AG052603)

\section{References}

1. Fieselmann JF, Hendryx MS, Helms CM, Wakefield DS. Respiratory rate predicts cardiopulmonary arrest for internal medicine inpatients. J Gen Intern Med. 1993;8(7):354-360. https://doi.org/10.1007/BF02600071.

2. Hodgetts TJ, Kenward G, Vlachonikolis IG, Payne S, Castle N. The identification of risk factors for cardiac arrest and formulation of activation criteria to alert a medical emergency team. Resuscitation. 2002;54(2):125-131. https:// doi.org/10.1016/S0300-9572(02)00100-4.

3. Bone RC, Sibbald WJ, Sprung CL. The ACCP-SCCM consensus conference on sepsis and organ failure. Chest. 1992;101(6):1481-1483.

4. Lovett PB, Buchwald JM, Sturmann K, Bijur P. The vexatious vital: neither clinical measurements by nurses nor an electronic monitor provides accurate measurements of respiratory rate in triage. Ann Emerg Med. 2005;45(1):6876. https://doi.org/10.1016/j.annemergmed.2004.06.016.

5. Chen J, Hillman K, Bellomo R, et al. The impact of introducing medical emergency team system on the documentations of vital signs. Resuscitation. 2009;80(1):35-43. https://doi.org/10.1016/j.resuscitation.2008.10.009.

6. Leuvan $\mathrm{CH}$, Mitchell I. Missed opportunities? An observational study of vital sign measurements. Crit Care Resusc. 2008;10(2):111-115.

7. Badawy J, Nguyen OK, Clark C, Halm EA, Makam AN. Is everyone really breathing 20 times a minute? Assessing epidemiology and variation in recorded respiratory rate in hospitalised adults. BMJ Qual Saf. 2017;26(10):832836. https://doi.org/10.1136/bmjqs-2017-006671.

8. Chua WL, Mackey S, Ng EK, Liaw SY. Front line nurses' experiences with deteriorating ward patients: a qualitative study. Int Nurs Rev. 2013;60(4):501509. https://doi.org/10.1111/inr.12061. 
9. De Meester K, Van Bogaert P, Clarke SP, Bossaert L. In-hospital mortality after serious adverse events on medical and surgical nursing units: a mixed methods study. J Clin Nurs. 2013;22(15-16):2308-2317. https://doi.org/10.1111/ j.1365-2702.2012.04154.x.

10. Cheng AC, Black JF, Buising KL. Respiratory rate: the neglected vital sign Med J Aust. 2008;189(9):531. https://doi.org/10.5694/j.1326-5377.2008 tb02163.x.

11. Mok W, Wang W, Cooper S, Ang EN, Liaw SY. Attitudes towards vital signs monitoring in the detection of clinical deterioration: scale development and survey of ward nurses. Int J Qual Health Care. 2015;27(3):207-213. https://doi. org/10.1093/intqhc/mzv019.

12. Keshvani N, Berger K, Nguyen OK, Makam AN. Roadmap for improving the accuracy of respiratory rate measurements. BMJ Qual Saf. 2018;27(8):e5. https://doi.org/10.1136/bmjgs-2017-007516.

13. Semler MW, Stover DG, Copland AP, et al. Flash mob research: a single-day, multicenter, resident-directed study of respiratory rate. Chest. 2013;143(6):1740-1744. https://doi.org/10.1378/chest.12-1837.

14. Makam AN, Nguyen OK, Auerbach AD. Diagnostic accuracy and effectiveness of automated electronic sepsis alert systems: a systematic review. J Hosp Med. 2015;10(6):396-402. https://doi.org/10.1002/jhm.2347.

15. Ploeg J, Skelly J, Rowan M, et al. The role of nursing best practice champions in diffusing practice guidelines: a mixed methods study. Worldviews Evid Based Nurs. 2010;7(4):238-251. https://doi.org/10.1111/j.17416787.2010.00202.x. 